\title{
Multifactor-dimensionality reduction shows a two-locus interaction associated with Type 2 diabetes mellitus
}

\author{
Y. M. Cho ${ }^{1,2}$ • M. D. Ritchie ${ }^{3}$ J. H. Moore ${ }^{3}$ J. Y. Park ${ }^{4}$ K.-U. Lee ${ }^{4}$ H. D. Shin ${ }^{5}$ H. K. Lee ${ }^{1}$ K. S. Park ${ }^{1,2}$ \\ ${ }^{1}$ Department of Internal Medicine, Seoul National University College of Medicine, Seoul, Korea \\ ${ }^{2}$ Genome Research Center for Diabetes and Endocrine Disease, Clinical Research Institute, Seoul National University Hospital, \\ Seoul, Korea \\ 3 Program in Human Genetics and Department of Molecular Physiology and Biophysics, Vanderbilt University Medical School, \\ Nashville, Tennessee, USA \\ ${ }^{4}$ Department of Internal Medicine, University of Ulsan School of Medicine, Seoul, Korea \\ ${ }^{5}$ Department of Genetic Epidemiology, SNP Genetics, Seoul, Korea
}

\section{Abstract}

Aims/hypothesis. Type 2 diabetes mellitus is a complex genetic disease, which results from interactions between multiple genes and environmental factors without any single factor having strong independent effects. This study was done to identify gene to gene interactions which could be associated with the risk of Type 2 diabetes.

Methods. We genotyped 23 different loci in the 15 candidate genes of Type 2 diabetes in 504 unrelated Type 2 diabetic patients and 133 non-diabetic control subjects. We analysed gene to gene interactions among 23 polymorphic loci using the multifactor-dimensionality reduction (MDR) method, which has been shown to be effective for detecting and characterising gene to gene interactions in case-control studies with relatively small samples.

Results. The MDR analysis showed a significant gene to gene interaction between the Ala55Val polymorphism in the uncoupling protein 2 gene (UCP2) and the $161 \mathrm{C}>\mathrm{T}$ polymorphism in the exon 6 of peroxisome proliferator-activated receptor $\gamma(P P A R \gamma)$ gene. This interaction showed the maximum consistency and minimum prediction error among all gene to gene interaction models evaluated. Moreover, the combination of the UCP2 $55 \mathrm{Ala} / \mathrm{Val}$ heterozygote and the PPAR $\gamma 161 \mathrm{C} / \mathrm{C}$ homozygote was associated with a reduced risk of Type 2 diabetes (odds ratio: $0.51,95 \%$ CI: 0.34 to $0.77, p=0.0016$ ).

Conclusions/interpretation. Using the MDR method, we showed a two-locus interaction between the UCP2 and PPAR $\gamma$ genes among 23 loci in the candidate genes of Type 2 diabetes. The determination of such genotype combinations contributing to Type 2 diabetes mellitus could provide a new tool for identifying high-risk individuals. [Diabetologia (2004) 47:549-554]

Keywords Multifactor-dimensionality reduction . Type 2 diabetes mellitus - Gene to gene interaction . Uncoupling protein 2 . Peroxisome proliferatoractivated receptor $\gamma$
Received: 30 June 2003 / Revised: 24 November 2003

Published online: 17 January 2004

(C) Springer-Verlag 2004

\section{K. S. Park (৫)}

Department of Internal Medicine,

Seoul National University College of Medicine,

28 Yongon-Dong Chongno-Gu, Seoul, 110-744 Korea

E-mail:kspark@snu.ac.kr

Abbreviations: MDR, Multifactor-dimensionality reduction SNP, single nucleotide polymorphism $\cdot \mathrm{CV}$, cross-validation * UCP, uncoupling protein - PPAR $\gamma$, peroxisome proliferatoractivated receptor $\gamma \cdot$ FABP, fatty acid binding protein . ADRB3, $\beta 3$-adrenergic receptor - IRS1, insulin receptor substrate $1 \cdot$ OR, odds ratio
Type 2 diabetes mellitus is a common complex genetic disease. Its high incidence in certain populations and among first-degree relatives of Type 2 diabetic patients, as well as the high concordance in identical twins, provides strong evidence that genetic factors underlie susceptibility to the disease [1]. Monogenic forms account for only five per cent or less of all cases of Type 2 diabetes [2], while the common form of the disease results from interactions between multiple genes and environmental factors, without any single factor having strong independent effects [3]. Thus, searching for susceptibility genes for the common form of Type 2 diabetes requires a thorough understanding of gene to gene and gene to environment interactions. 
Traditionally, gene to gene interactions in complex diseases have been examined by logistic regression, multilocus linkage disequilibrium tests and the HardyWeinberg equilibrium test, all of which have limitations in their general application [4]. Thus, the identification and characterisation of gene to gene interactions has been limited mainly by a lack of powerful statistical methods and a lack of large sample size [4]. To overcome these limitations, the multifactor-dimensionality reduction (MDR) method was developed. It is used for detecting and characterising high-order gene to gene interactions in case-control and discordant-sib-pair studies with relatively small samples [5]. The MDR defines a single variable that incorporates information from several loci and/or incorporates environmental factors that can be divided into high-risk and low-risk combinations. This new variable can be evaluated for its ability to classify and predict disease risk status using cross-validation (CV) and permutation testing. It can also be used in conventional analytic methods [4]. The MDR method has been shown to have good power in relatively small case-control studies $[6,7]$.

In this study, we genotyped 23 different loci in the 15 candidate genes of Type 2 diabetes in unrelated Type 2 diabetic patients and non-diabetic control subjects, and examined gene to gene interactions with the MDR method.

\section{Subjects and methods}

Subjects. We studied 504 unrelated patients with Type 2 diabe-

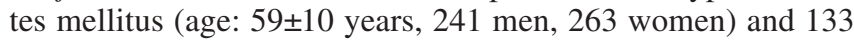
non-diabetic control subjects (age: $65 \pm 3$ years, 40 men, 93 women). Type 2 diabetes was diagnosed according to World Health Organization criteria [8]. Selection of the non-diabetic control subjects was according to the following criteria: 60 years or older, no past history of diabetes, no diabetes in firstdegree relatives, a fasting plasma glucose concentration of less than $6.1 \mathrm{mmol} / \mathrm{l}$, and an $\mathrm{HbA}_{1 \mathrm{c}}$ value of less than $5.8 \%$. The Institutional Review Board of Clinical Research Institute in Seoul National University Hospital, Korea approved the study protocol and informed consent for genetic analysis was obtained from each subject. This study was carried out in accordance with the Declaration of Helsinki as revised in 2000 (http://www.wma.net/e/policy/17cnote.pdf).

Selection of polymorphisms. Using public databases, e.g. PubMed and Online Mendelian Inheritance in Man (http://www. ncbi.nlm.nih.gov/Omim/), we selected 15 candidate genes that have been characterised and potentially associated with Type 2 diabetes. Among these candidate genes, calpain 10 and adiponectin genes (located in 2q37.3 and 3q27 respectively) are positional candidates evidenced by genome-wide linkage analysis $[9,10]$. In addition, peroxisome proliferator-activated receptor $\gamma(P P A R \gamma)$, fatty acid binding protein $(F A B P) 2, \beta 3$-adrenergic receptor $(A D R B 3)$ and insulin receptor substrate 1 (IRS1) have been linked to the phenotypes of Type 2 diabetes, e.g. obesity or insulin resistance [11, 12, 13, 14]. GenBank accession numbers for genes studied in this article are as follows: uncoupling protein $(U C P) 2$ (NM_003355), adiponectin
(NM_004797), PPAR $\gamma$ (NM_015869), FABP2 (NM_000134), PPAR $\gamma$ coactivator 1 (NM_013261), FABP3 (NM_004102), uncoupling protein 3 (NM_003356), calpain 10 (NM_021251), resistin (NM_020415), mitochondrial NADH dehydrogenase subunit 2 (J01415), mitochondrial ATPase 8 gene (J01415), mitochondrial D-loop (J01415), ADRB3 (NM_000025), IRS1 (NM_005544), and glycogen synthase 1 (NM_002103).

We further selected 23 polymorphisms, mostly single nucleotide polymorphisms (SNPs), within these genes. The 23 polymorphisms examined in this study are listed in Table 1. The minus signs before the numbered nucleotide in some polymorphisms, such as -345 in Table 1 , refer to the $5^{\prime}$ upstream region relative to the transcription-initiation site of a gene. To identify the polymorphisms in $F A B P 3$ gene, we sequenced the full gene, including -1500 bp promoter region, with 24 Korean DNA samples using ABI PRISM 3700 DNA analyser (Applied Biosystems, Foster City, Calif., USA). As a result, 14 polymorphisms were identified: 2 ins/dels, 2 short tandem repeats and 10 SNPs [15]. Among identified polymorphisms, -345T $>$ C, $+5428 \mathrm{G}>\mathrm{C},+7836 \mathrm{~T}$ ins $>$ del, and $-530 \mathrm{CTC}$ ins $>$ del were included in this study.

Genotyping of polymorphisms. Genomic DNA was isolated with a commercial kit (Gentra Systems, Minneapolis, Minn., USA). The amplifying and extension primers were designed for single base extension for SNPs and ins/del. Primer extension reactions were done with SNaPshot ddNTP Primer Extension Kit (Applied Biosystems). To clean up the primer extension reaction, one unit of shrimp alkaline phosphatase was added to the reaction mixture, which was then incubated for $1 \mathrm{~h}$ at $37^{\circ} \mathrm{C}$, followed by $15 \mathrm{~min}$ at $72^{\circ} \mathrm{C}$. The DNA samples containing extension products and GeneScan 120 Liz size standard solution were added to Hi-Di Formamide (Applied Biosystems) according to the manufacturer's instructions. The mixture was incubated for $5 \mathrm{~min}$ at $95^{\circ} \mathrm{C}$ followed by $5 \mathrm{~min}$ on ice, and then was electrophoresed by ABI Prism 3100 Genetic Analyzer. Automatic genotype assignment was done by ABI Prism GeneScan and Genotyper software (Applied Biosystems).

Multifactor-dimensionality reduction analysis. MDR was done as described previously [4, 5, 6, 7]. Briefly, the data set is divided into ten parts of equal size for 10 -fold CV. A training set composed of $9 / 10$ of the data used to build the MDR model is selected. The remaining $1 / 10$ of the data is used to compose the testing set, which is used to determine the generalisability of the MDR model. Next, a set of $n$ genetic and/or environmental factors is selected. The $n$ factors are represented in $n$-dimensional space. During the model selection process, each multifactor cell class in $n$-dimensional space is labelled as high-risk if the ratio of cases to controls meets or exceeds the threshold of 1.0 and as low-risk if the threshold is not exceeded. We have observed in simulation studies that a threshold of 1.0 is optimal at this stage of MDR modelling for unbalanced data sets (unpublished results by the authors).

When the final best model is selected, a model for high-risk and low-risk genotype combinations is formed using an adjusted threshold that is equal to the ratio of cases and controls in the dataset. Among all of the two-factor combinations, a single model that maximises the cases to controls ratio is selected. This two-locus model has the minimum classification error among the two-locus models. To evaluate the predictive ability of the model, the model is evaluated on the basis of the 1/10 of the data left out for testing. This procedure is done ten times, each time using a different $1 / 10$ of the data for testing. In our study we also performed this entire ten-fold CV procedure ten times, each time using different random number seeds to re- 
Table 1. Allele frequency of each polymorphism

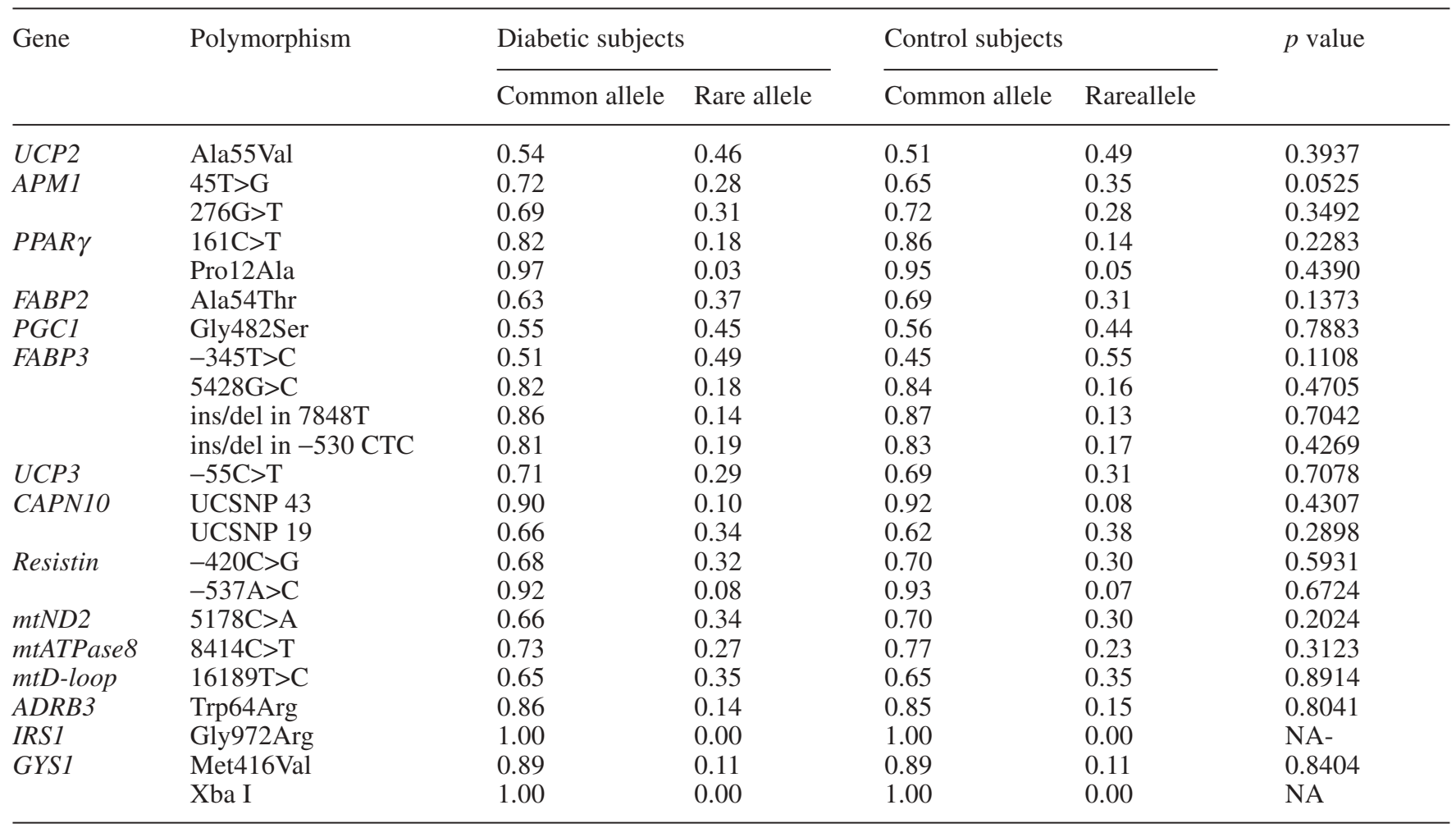

$U C P 2$, uncoupling protein 2; $A P M 1$, adiponectin; $P P A R \gamma$, peroxisome proliferator-activated receptor gamma; $F A B P 2$, fatty acid binding protein $2 ; P G C 1$, peroxisome proliferator-activated receptor gamma coactivator $1 ; F A B P 3$, fatty acid binding protein $3 ; U C P 3$, uncoupling protein 3 ; CAPN10, calpain
10; $m t N D 2$, mitochondrial NADH dehydrogenase subunit 2; mtATPase 8 , mitochondrial ATPase 8 ; $m t D$-loop, mitochondrial D-loop (control region); $A D R B 3, \beta 3$-adrenergic receptor; IRS1, insulin receptor substrate 1; GYS1, glycogen synthase 1; NA, not applicable duce the chance of observing spurious results due to chance divisions of the data.

This MDR procedure can be carried out for each possible model size (i.e. each number of loci and/or environmental factors), if computationally feasible. Due to computation restrictions, we set out to detect all two-locus interactions through five-locus interactions. The result is a set of models, one for each model size considered. From this set, the model with the combination of loci and/or discrete environmental factors that maximises the $\mathrm{CV}$ consistency and minimises the prediction error is selected. Cross-validation consistency is a measure of the number of times a particular set of loci and/or factors are identified in each possible $9 / 10$ of the subjects $[5,6,7]$. The proportion of subjects for whom an incorrect prediction was made is the prediction error. When CV consistency is maximal for one model and prediction error is minimal for another, statistical parsimony is used to choose the best model. Thus when the $\mathrm{CV}$ metric and the prediction error metric support different models, the model with the fewest loci/factors is selected. Hypothesis testing of this final best model can then be done by evaluating the magnitude of the prediction error. We determined statistical significance by comparing the average prediction error from the observed data with the distribution of average prediction errors under the null hypothesis of no associations derived empirically from 100 permutations. In this study, prediction error is evaluated for the best model identified by MDR in each permuted dataset. The null hypothesis was rejected when the upper-tail Monte Carlo $p$ value derived from the permutation test was 0.05 or lower.
Statistical analysis. Allele frequencies were estimated by genecounting method, and the chi-square test was used to examine the Hardy-Weinberg equilibrium. Each genotype was assessed by chi square test with the use of dominant, recessive and additive genetic models. To examine the results of the MDR analysis, logistic regression analysis was used and odds ratios (OR) with $95 \%$ CIs were obtained. A $p$ value of less than 0.05 was considered statistically significant.

\section{Results}

Allele frequencies and association with Type 2 diabetes mellitus. All 23 polymorphisms examined in this study were in Hardy-Weinberg equilibrium and their allele frequencies were similar in the patients with Type 2 diabetes and in the control subjects (Table 1). Among the results of the association study using the dominant, recessive and additive genetic models, no locus was found to be associated with Type 2 diabetes after correction for multiple comparisons.

MDR analysis. Table 2 summarises, for each number of loci evaluated, the average CV consistency and average prediction error obtained from MDR analysis of the data set of subjects with and without Type 2 di- 
Table 2. Summary of MDR results

\begin{tabular}{llll}
\hline No. of loci in model & Genes included in the best combination in each modela & Cross-validation consistency & Prediction error \\
\hline $\mathbf{2}$ & $U C P 2, P P A R \gamma$ & $\mathbf{8 . 6 0}$ & $\mathbf{2 0 . 4 3}$ b \\
3 & $U C P 2, P P A R \gamma, F A B P 3 a$ & 4.14 & 21.91 \\
4 & $A P M 1, P P A R \gamma, F A B P 3 b, C A P N 10$ & 5.20 & 22.96 \\
5 & $U C P 2, F A B P 2, P G C 1, F A B P 3 b, C A P N 10$ & 4.67 & 28.46 \\
\hline
\end{tabular}

The multilocus model with maximum cross-validation consistency and minimum prediction error is indicated in bold type a $U C P 2$, UCP2 Ala55Val polymorphism; PPAR $\gamma$, PPAR $\gamma$ $161 \mathrm{C}>\mathrm{T}$ polymorphism; FABP3a, FABP3 $5428 \mathrm{G}>\mathrm{C}$ polymorphism; $A P M 1$, adiponectin $45 \mathrm{~T}>\mathrm{G}$ polymorphism; $F A B P 3 b$,

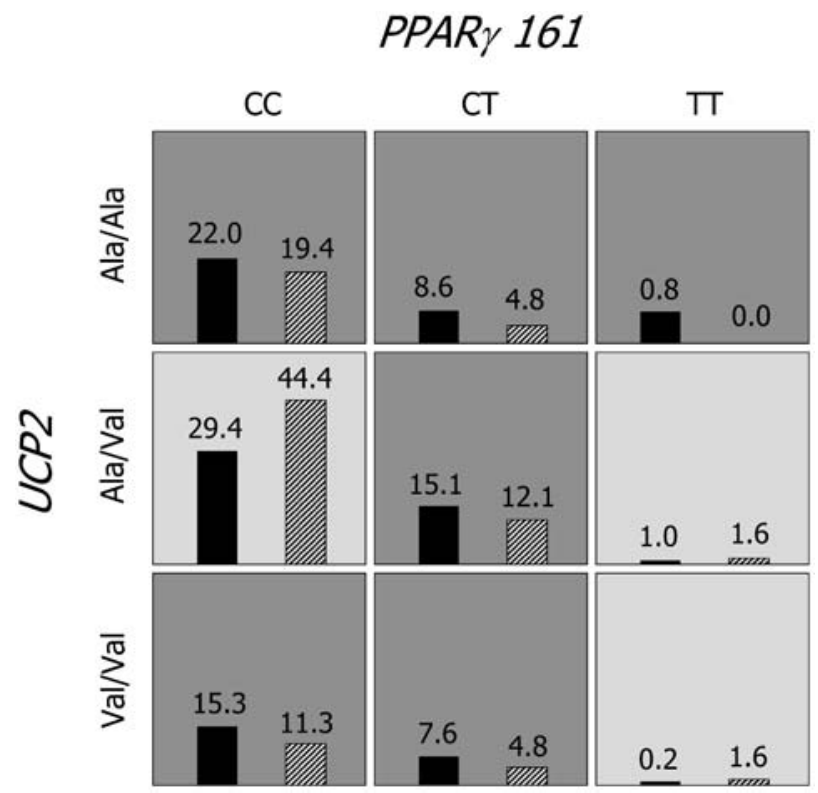

Fig. 1. Distribution of high-risk and low-risk genotypes in the best two-locus model. This summary of the distribution shows high-risk (dark shading) and low-risk (light shading) genotypes associated with Type 2 diabetes mellitus in the two-locus interaction detected by MDR analysis. The percentage of diabetic subjects (left black bar in boxes) and control subjects (right hatched bar in boxes) is shown for each two-locus genotype combination. Boxes were labelled as high-risk if the ratio of the percentage of cases to controls met or exceeded the threshold of 1.0 (the ratio of the total number, not the percentage, of cases to the total number of controls in the dataset was 3.789). Boxes were labelled as low-risk if the threshold was not exceeded. Based on the pattern of high-risk and low-risk genotypes, this two-locus model is evidence of gene to gene interaction

abetes. One two-locus model had a minimum prediction error of $20.43 \%(p=0.01)$ and a maximum CV consistency of 8.6 out of 10. This two-locus model, which included the Ala55Val polymorphism in the $U C P 2$ gene and the $161 \mathrm{C}>\mathrm{T}$ polymorphism in exon 6 of the PPAR $\gamma$ gene (Fig. 1), was regarded as the best model.

Conventional statistical analyses. With regard to the question of whether the genotype combinations from
FABP3 -345T>C polymorphism; CAPN10, UCSNP19 of the calpain 10 gene; FABP2, FABP2 Ala45Thr polymorphism; $P G C 1$, PGC1 Gly482Ser polymorphism

b $p=0.01$

the UCP2 Ala55Val polymorphism and the PPAR $\gamma$ $161 \mathrm{C}>\mathrm{T}$ polymorphism are associated with susceptibility to Type 2 diabetes, the overall chi square of all the genotype combinations of UCP2 Ala55Val and $P P A R \gamma 161 \mathrm{C} / \mathrm{C}$ was $8.805(d f=4, p=0.0030)$. As genotype combinations with PPAR $\gamma$ 161T/T homozygote are very rare (as shown in Fig. 1), it is difficult to classify these combinations into high- or low-risk groups. Excluding the rare genotype combinations harbouring PPAR $\gamma$ 161T/T homozygote, the combination of UCP2 55Ala/Val and PPAR $\gamma$ 161C/C was the low-risk combination, while all others corresponded to the highrisk combination. In this model, the OR for the lowrisk combination of the $U C P 255 \mathrm{Ala} / \mathrm{Val}$ heterozygote and the PPAR $161 \mathrm{C} / \mathrm{C}$ homozygote was $0.51(95 \%$ CI: 0.34 to $0.77, p=0.0016$ ).

\section{Discussion}

Using the MDR method followed by conventional statistical analysis, we showed an interaction between two of 23 loci in 15 candidate genes of Type 2 diabetes. It is well known that Type 2 diabetes is polygenic or multifactorial in nature but the gene to gene interactions associated with the disease have been very difficult to demonstrate. This obstacle has been mainly explained by the lack of powerful statistical methods and lack of large sample sizes [4]. In this study, we effectively found evidence for a significant gene to gene interaction among two loci out of a total of 23 loci, proving the MDR method used by us to be effective in detecting multigenic interactions among many different loci in many different genes.

Using only two candidate SNPs, several studies have shown gene to gene interactions in obesity and insulin resistance but not in Type 2 diabetes. The $A D R B 3$ gene has been the gene most frequently examined and reported in connection with gene to gene interaction, e.g. with $U C P 1$ gene in Danish [16] and in Finnish populations [17], with the human type 2 deiodinase gene in white populations [18], with the FABP2 gene in Japanese [19] and with the PPAR $\gamma 2$ genes in Mexican American populations [20]. In a different 
way, using multi-point allele-sharing analysis with lod score, other investigators showed that the loci on chromosome 2 (NIDDMI) and 15 (near CYP19) interact to increase susceptibility to Type 2 diabetes in Mexican Americans [21].

Among several models with different numbers of loci, the best gene to gene interaction model identified in our study from 23 loci of 15 candidate genes was a two-locus model including the UCP2 Ala55Val polymorphism and the PPAR $\gamma 161 \mathrm{C}>\mathrm{T}$ polymorphism. In this model, the combination of the UCP2 $55 \mathrm{Ala} / \mathrm{Val}$ heterozygote and the PPAR $\gamma 161 \mathrm{C} / \mathrm{C}$ homozygote was associated with a reduced risk of Type 2 diabetes. Although these variants have been studied for their association with susceptibility to Type 2 diabetes in several populations [22, 23, 24], a significant association with Type 2 diabetes has not been reported independently for each variant. In this study, by contrast, the combined effect of these variants was a significantly reduced risk of Type 2 diabetes, which implies a genetic interaction between these genes in the pathogenesis of the disease.

However, the nature of the gene to gene interaction between the UCP2 Ala55Val polymorphism and the PPAR $\gamma 161 \mathrm{C}>\mathrm{T}$ polymorphism is not clear, as suggested previously when the MDR method was applied to breast cancer [5]. If there was a consistent trend of high-risk or low-risk cells across a series of rows or of columns, it could indicate that a particular locus had a main effect. We could not find such a trend in our best two-locus model. As Type 2 diabetes does not have a typical pattern of Mendelian inheritance that can be explained by a linear model, our observation could be regarded as quite natural. Similarly, other investigators reported that the greatest risk of Type 2 diabetes in Mexican Americans was associated with the 112/121-haplotype combination in CAPN10 gene, but they were unable to find an increased risk of Type 2 diabetes in subjects who were homozygous for either of the haplotypes [9].

Although Type 2 diabetes is a heterogeneous syndrome resulting from defects of insulin secretion and insulin action [25], we mainly included the genes responsible for insulin resistance in this study. A balanced number of genes responsible for both insulin secretion and insulin action was not studied. Given gene to gene interactions are expected to be a ubiquitous component of the genetic architecture of common human diseases such as Type 2 diabetes mellitus [26], and the identification, by MDR, of genotype combinations contributing to Type 2 diabetes could enable timely identification of high-risk individuals, who could benefit from early behavioral or medical intervention to prevent the disease developing.

Acknowledgements. This study was supported by a grant from the Korea Health 21 R \& D Project, Ministry of Health and Welfare, Republic of Korea (00-PJ3-PG6-GN07-001).

\section{References}

1. Elbein SC (2002) Perspective: the search for genes for type 2 diabetes in the post-genome era. Endocrinology 143:2012-2018

2. Permutt MA, Hattersley AT (2000) Searching for type 2 diabetes genes in the post-genome era. Trends Endocrinol Metab 11:383-393

3. Busch CP, Hegele RA (2001) Genetic determinants of type 2 diabetes mellitus. Clin Genet 60:243-254

4. Moore JH, Williams SM (2002) New strategies for identifying gene-gene interactions in hypertension. Ann Med 34:88-95

5. Ritchie MD, Hahn LW, Roodi N et al. (2001) Multifactordimensionality reduction reveals high-order interactions among estrogen-metabolism genes in sporadic breast cancer. Am J Hum Genet 69:138-147

6. Ritchie MD, Hahn LW, Moore JH (2003) Power of multifactor dimensionality reduction for detecting gene-gene interactions in the presence of genotyping error, missing data, phenocopy, and genetic heterogeneity. Genet Epidemiol 24:150-157

7. Hahn LW, Ritchie MD, Moore JH (2003) Multifactor dimensionality reduction software for detecting gene-gene and gene-environment interactions. Bioinformatics 19:376382

8. Alberti KG, Zimmet PZ (1998) Definition, diagnosis and classification of diabetes mellitus and its complications. Part 1: diagnosis and classification of diabetes mellitus provisional report of a WHO consultation. Diabet Med 15:539-553

9. Horikawa Y, Oda N, Cox NJ et al. (2000) Genetic variation in the gene encoding calpain-10 is associated with type 2 diabetes mellitus. Nat Genet 26:163-175

10. Vionnet N, Hani El-H, Dupont S et al. (2000) Genomewide search for type 2 diabetes-susceptibility genes in French whites: evidence for a novel susceptibility locus for earlyonset diabetes on chromosome 3q27-qter and independent replication of a type 2-diabetes locus on chromosome 1q21-q24. Am J Hum Genet 67:1470-1480

11. Hsueh WC, Mitchell BD, Schneider JL et al. (2001) Genome-wide scan of obesity in the Old Order Amish. J Clin Endocrinol Metab 86:1199-1205

12. Prochazka M, Lillioja S, Tait JF et al (1993) Linkage of chromosomal markers on $4 \mathrm{q}$ with a putative gene determining maximal insulin action in Pima Indians. Diabetes 42:514-519

13. Mitchell BD, Cole SA, Comuzzie AG et al. (1999) A quantitative trait locus influencing BMI maps to the region of the beta-3 adrenergic receptor. Diabetes 48:18631867

14. Rice T, Chagnon YC, Perusse L et al. (2002) A genomewide linkage scan for abdominal subcutaneous and visceral fat in black and white families: The HERITAGE Family Study. Diabetes 51:848-855

15. Shin HD, Kim LH, Park BL et al. (2003) Polymorphisms in fatty acid-binding protein-3 (FABP3)-putative association with type 2 diabetes mellitus. Hum Mutat 22:180 (Abstract)

16. Urhammer SA, Hansen T, Borch-Johnsen K, Pedersen $\mathrm{O}$ (2000) Studies of the synergistic effect of the Trp/Arg64 polymorphism of the beta3-adrenergic receptor gene and the $-3826 \mathrm{~A} \rightarrow \mathrm{G}$ variant of the uncoupling protein-1 gene on features of obesity and insulin resistance in a population-based sample of 379 young Danish subjects. J Clin Endocrinol Metab 85:3151-3154 
17. Sivenius K, Valve R, Lindi V, Niskanen L, Laakso M, Uusitupa M (2000) Synergistic effect of polymorphisms in uncoupling protein 1 and beta3-adrenergic receptor genes on long-term body weight change in Finnish type 2 diabetic and non-diabetic control subjects. Int $\mathrm{J}$ Obes Relat Metab Disord 24:514-519

18. Mentuccia D, Proietti-Pannunzi L, Tanner K et al. (2002) Association between a novel variant of the human type 2 deiodinase gene Thr92Ala and insulin resistance: evidence of interaction with the Trp64Arg variant of the beta-3adrenergic receptor. Diabetes 51:880-883

19. Ishii T, Hirose H, Kawai T et al. (2001) Effects of intestinal fatty acid-binding protein gene Ala54Thr polymorphism and beta3-adrenergic receptor gene Trp64Arg polymorphism on insulin resistance and fasting plasma glucose in young to older Japanese men. Metabolism 50:13011307

20. Hsueh WC, Cole SA, Shuldiner AR et al. (2001) Interactions between variants in the beta3-adrenergic receptor and peroxisome proliferator-activated receptor-gamma2 genes and obesity. Diabetes Care 24:672-677
21. Cox NJ, Frigge M, Nicolae DL et al. (1999) Loci on chromosomes 2 (NIDDM1) and 15 interact to increase susceptibility to diabetes in Mexican Americans. Nat Genet 21:213-215

22. Dalgaard LT, Pedersen O (2001) Uncoupling proteins: functional characteristics and role in the pathogenesis of obesity and Type II diabetes. Diabetologia 44:946-965

23. Meirhaeghe A, Fajas L, Helbecque N et al. (1998) A genetic polymorphism of the peroxisome proliferator-activated receptor gamma gene influences plasma leptin levels in obese humans. Hum Mol Genet 7:435-440

24. Poulsen P, Andersen G., Fenger M et al. (2003) Impact of two common polymorphisms in the PPARgamma gene on glucose tolerance and plasma insulin profiles in monozygotic and dizygotic twins: thrifty genotype, thrifty phenotype, or both? Diabetes 52:194-198

25. DeFronzo RA (1988) Lilly lecture 1987. The triumvirate: beta-cell, muscle, liver. A collusion responsible for NIDDM. Diabetes 37:667-687

26. Moore JH (2003) The ubiquitous nature of epistasis in determining susceptibility to common human diseases. Hum Hered 56:73-82 